\title{
Virtual Prototype of Innovative Ka-Band Power Amplifier Based on Waveguide Polarizer
}

\author{
Stefano Fantauzzi ${ }^{1}$, Lorenzo Valletti ${ }^{1}$ and Franco Di Paolo' \\ ${ }^{1}$ University of Roma "Tor Vergata", via del Politecnico, 1, Roma, Italy \\ Corresponding author: (e-mail: stefano.fantauzzi@alumni.uniroma2.eu).
}

\begin{abstract}
This paper outlines an innovative approach to design a spatial power-combining structure based on waveguide polarizers. It presents the 3D CAD model of the new structure with the transversal probes and considerations in positioning and optimization of them. Exploiting the transformation of the dominant input mode $\mathrm{TE}_{10}$ into an elliptically polarized wave, provided by the polarizer, it has been possible to achieve a division of power by eight, completely carried out in space. With the insertion of the transversal probes made by microstrips, the RF signal can be sent to the MMIC solid state power amplifiers, and then recombined in the output section. Thanks to the large number of power divisions made in the waveguide section, the insertion loss of the power divider/combiner is less than $0.5 \mathrm{~dB}$ across the $32-34$ $\mathrm{GHz}$ band, achieving a great power density as well. At the authors' best knowledge, this is the first work where a waveguide polarizer is used in Spatial Power Combining technology.
\end{abstract}

INDEX TERMS SPC, Spatial Power Combiners, Power Amplifiers, MMIC, Waveguide Polarizers.

\section{INTRODUCTION}

$\mathbf{P}$ OWER dividers based on waveguide transitions are great candidates to be used in millimeter-wave electronic systems, both because of low insertion loss and high-power efficiency; they can effectively replace highpower vacuum tubes, providing a low weight structure, operating at low voltages with high reliability [1]. Several analytical models and topologies of SPC (spatial power combiners) have been studied in the last decade [2-6], that clearly show the immense potential of these architectures. The basic working principle of SPC technique lies in the power dividing/combining operation, performed in a singlestage parallel way; in case of waveguide systems, this is done in air, so the dissipation factor is very low [7]. The most interesting fact is that the combination losses are independent to the number of solid state amplifiers, further allowing for the combination of odd number of devices. Along with the power conditioning architectures, two major electromagnetic manipulation techniques were involved. The first one allows to obtain an orthogonal polarization traveling in the waveguide, by means of a waveguidepolarizer with inner corner ridges [8]; the second one implies the insertion of several probes across the waveguide section, which require a fixed back-short properly positioned $[9,10]$. Another milestone reached in this project is the large number of probes inserted into the waveguide. Up to now, a maximum of 4 transversal probes have been inserted in a waveguide structure [11], either coaxial or microstrip ones. Note that in that case, the waveguide used is a WR90, so the dimensions do not hinder the realization process too much. For this SPC though, the waveguide required is a WR28 (with internal dimensions of $7.112 \mathrm{~mm}$ $\mathrm{x} 3.556 \mathrm{~mm}$ ), a much smaller than the previous one. Moreover, the probes engaged in this project are made by microstrips, so that the complete structure is fully integrated.

In this work a novel structure for these kind of devices is presented. Starting from the idea of obtaining two independent dominant modes in the waveguide, which can be achieved by using a Ka-band waveguide polarizer, then the traveling power was captured using 8 transversal probes with their relative $\lambda / 4$ short metallic plate. Finally, the collected power was sent to the solid-state power amplifiers and recombined, in a symmetrical structure, to the output port in WR28 waveguide, same size of the input port. A practical knowledge of this kind of systems has allowed to optimize the distance between two probes lying on the same face of the square waveguide, making it possible to achieve a very high power-combining efficiency and an adequate heat sinking for the integrated amplifiers (MMIC). The operating frequency band of this new power amplifier/divider structure lies between 32-34 GHz.

This paper is organized as follows: in section II the design of the sub-parts of the system is discussed, in section III some simulated results are shown, section IV concerns the designed complete structure while in section $\mathrm{V}$ multiphysics analysis is presented, and finally in section VI the conclusions of the present work are summarized. 


\section{DESIGN}

The Design of the whole structure is divided into three parts, as follows:

\section{A. WAVEGUIDE POLARIZER}

Essentially this part is about 3D modeling of the structure studied in [8]. In that paper there are three optimized bands in which the polarizer works $(26-30 \mathrm{GHz}, 30-34 \mathrm{GHz}, 34-38$ $\mathrm{GHz}$ ). For this project the second bandwidth was considered, so a $30-34 \mathrm{GHz}$ waveguide polarizer was simulated and further optimized using HFSS Release 2019 [12]. To notice as the section of length $l s q$ (a parameter used in [8]) was cut off from the original structure, because it was too short in length and practically non realizable.
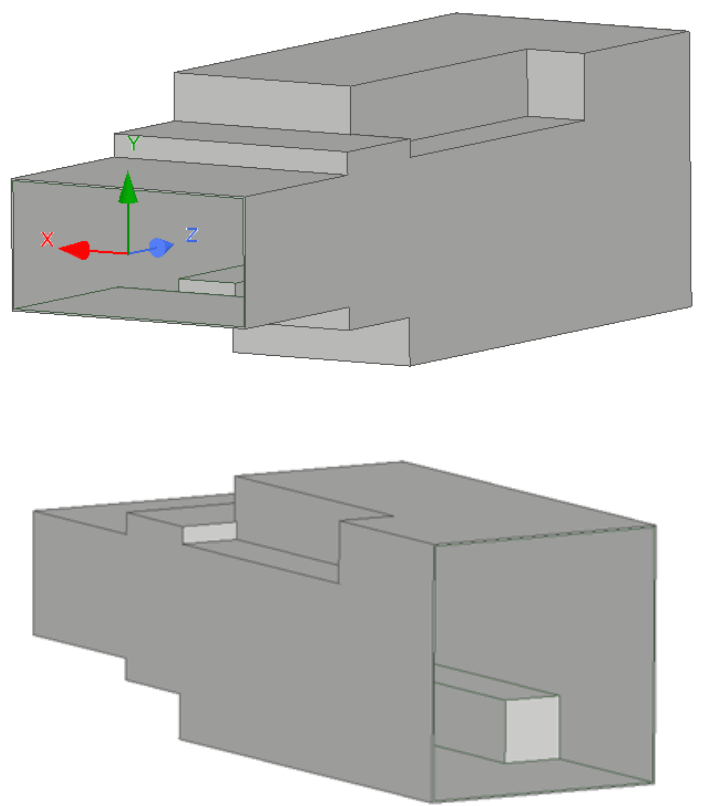

FIGURE 1. HFSS 3D Model of the Waveguide Polarizer.

\section{B. TRANSVERSAL PROBES}

Most of SPC architectures exploit longitudinal probes, such as finlines, in order to convey the electromagnetic power toward the MMIC amplifiers. For such a structure a transverse capture of the field was implemented, using eight independent probes made by microstrips, equally arranged in pairs on each face of the square waveguide. The most important measure to consider, is the penetration depth of the microstrips into the waveguide. Because of the angle that relate the orthogonal modes and the fundamental input mode (twist angle), they cannot share the same length but must follow the rotation of the $\mathrm{TE}_{10}$ mode. For this configuration, the penetration distance of the two probes, placed on a single face, is respectively of $1.2 \mathrm{~mm}$ and $1.7 \mathrm{~mm}$.

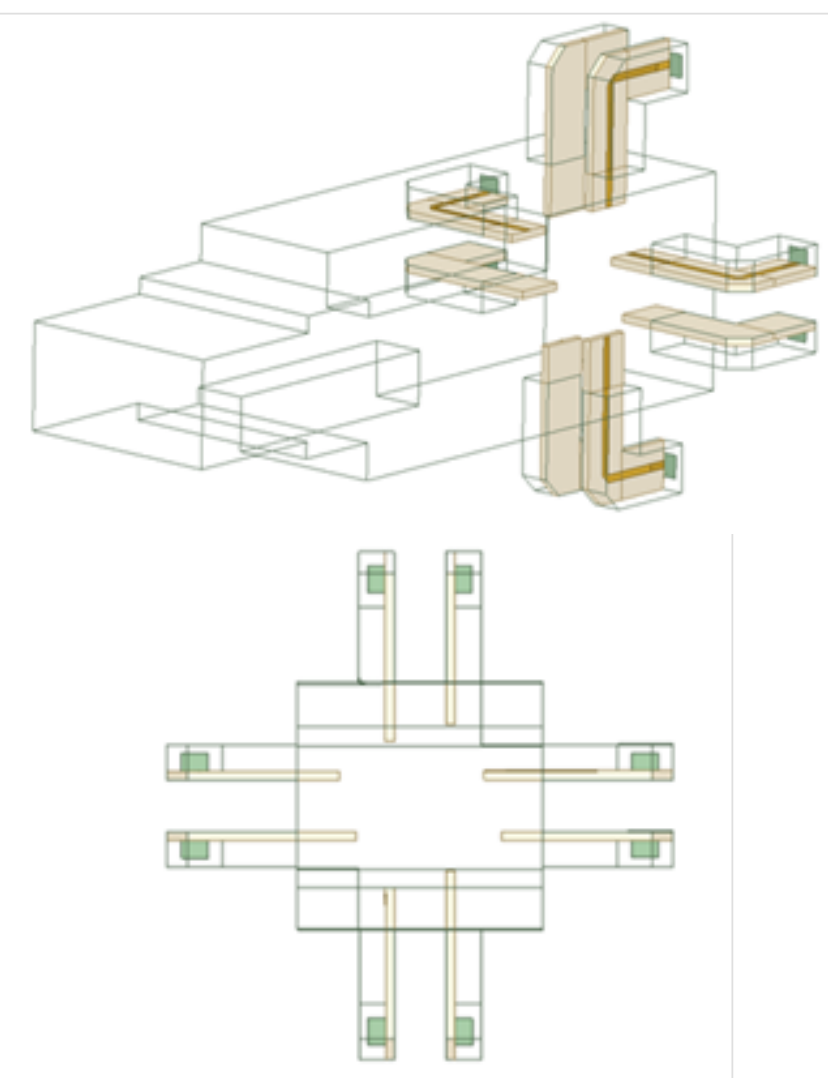

FIGURE 2. Transversal Probes Arrangement

Regarding the metallic back-short, this one was designed with respect to the central frequency of the optimized bandwidth $(32 \mathrm{GHz})$ and then is situated $2.34 \mathrm{~mm}(\lambda / 4)$ away from the hot plate of the microstrip.

\section{DIVIDER BY 8 BACK TO BACK}

At this point it is possible to mirror the structure designed so far in order to obtain the complete passive section of the SPC. This configuration, in fact, lacks the MMIC power amplifiers, then an analysis between input and output rectangular waveguide provides information about the scattering parameters of the passive structure. The back-toback configuration was obtained by mirroring along the $y$ axis the structure depicted in figure 2 , and it is shown in the following picture.

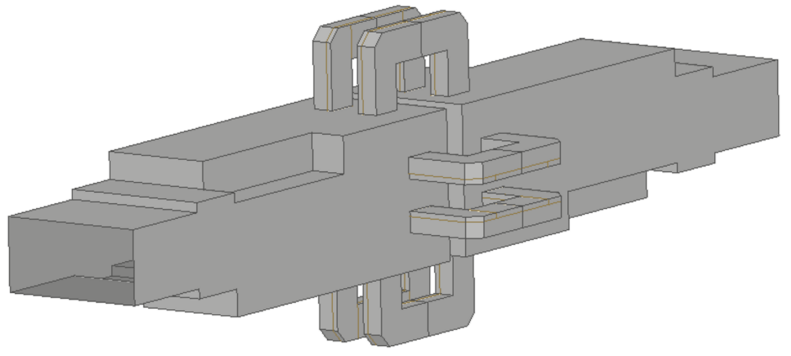

FIGURE 3. Back to back configuration. 


\section{SIMULATED RESULTS}

\section{A. WAVEGUIDE POLARIZER}

The basic parameters to focus on are the return loss $\left(\mathrm{S}_{11}\right)$, which must remain below the value $-10 \mathrm{~dB}$ in the working bandwidth and the power division between the fundamental ways $\mathrm{TE}_{10}$ and $\mathrm{TE}_{01}$ in the square section $[13,14]$.

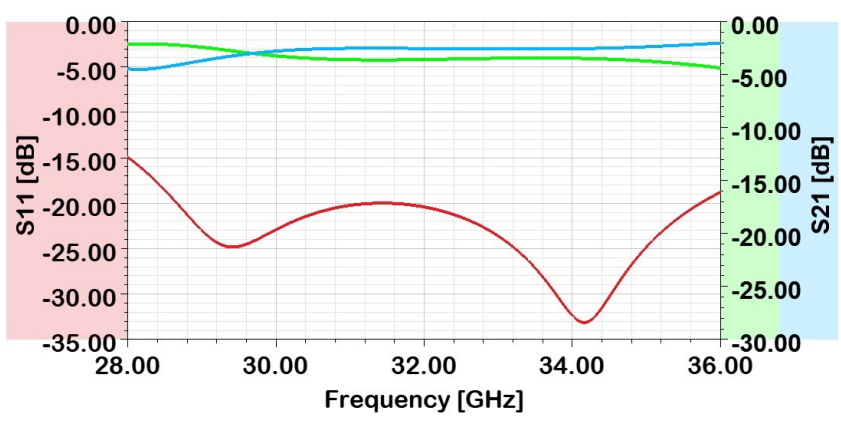

FIGURE 4. $S_{11}$ (red) and $S_{21}$ (green, blue) of the Polarizer

As well as the power modules, it is vital to also consider two other quantities which relate the input fundamental mode with the two orthogonal modes in the square section, and so, two angles must be measured. The first angle relates the position of the fundamental input mode TE10 on the xy plane, with the position of the mode $\mathrm{TE}_{10}$ on the same plane but located on the square section, then on the "output" port of the polarizer. This angle was called twist angle and must be as close as possible to the value of $0^{\circ}$ in the useful bandwidth. The other angle reports the phase shift between the two fundamental modes of the square waveguide, so the TE 10 and the $\mathrm{TE}_{01}$. As it is to understand, this angle should remain close to the value of $90^{\circ}$ and it is called Cross-Polarization angle.

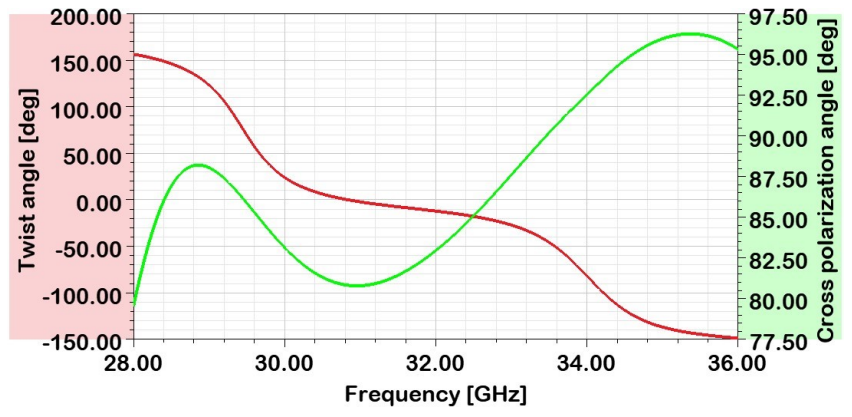

FIGURE 5. Twist (red) and Cross-Polarization (green) Angle

\section{B. TRANSVERSAL PROBES}

For this particular SPC system, a transverse field capturing technique was implemented, by means of microstrips probes and a metallic back-short [15]. The proper design of the probes disposition allowed to obtain an acceptable power division in the range of $30-34 \mathrm{GHz}$; we are talking about a value of $-9 \pm 1 \mathrm{~dB}$, a reasonable value in order to consider all the eight power amplifiers almost equally excited. The pattern of the $S_{i j}$ scattering parameters contains the information about the power division.

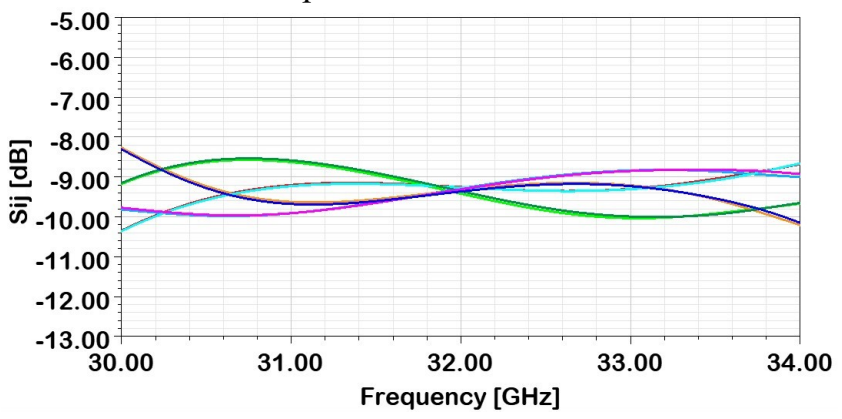

FIGURE 6. Power Division on the eight ports

\section{DIVIDER BY 8 BACK TO BACK}

The electromagnetic analysis of the back to back configuration is particularly useful in order to test the final matching of the structure, also verifying that no oscillations are triggered [16]. By imposing a stimulus, on either input and output port (of the structure depicted in figure 3), that agrees with the spatial arrangement of the fondamental mode $\mathrm{TE}_{10}$, a scattering parameteres analysis of the complete passive system was performed. Focusing on input matching and transmission losses, it is easy to observe how the system introduces an insertion loss less than $1 \mathrm{~dB}$ between input and output port, hence the actual loss before and after the solid state power amplifiers falls below $0.5 \mathrm{~dB}$. Within the 32-34 $\mathrm{GHz}$, either $\mathrm{S}_{11}$ and $\mathrm{S}_{21}$ remain well below the limits imposed in the project but, expanding the analysis to the whole bandwith covered by the WR28 waveguide, a resonant behavior emerges. This aspect severly limits the useful bandwith of the spatial power combiner but it can be, at least in part, attenuated; the most immediate technique to do this is to insert eco-absorbent material in some specific parts of the waveguide, for instance, right above the microstrip in the outer section of the waveguide.

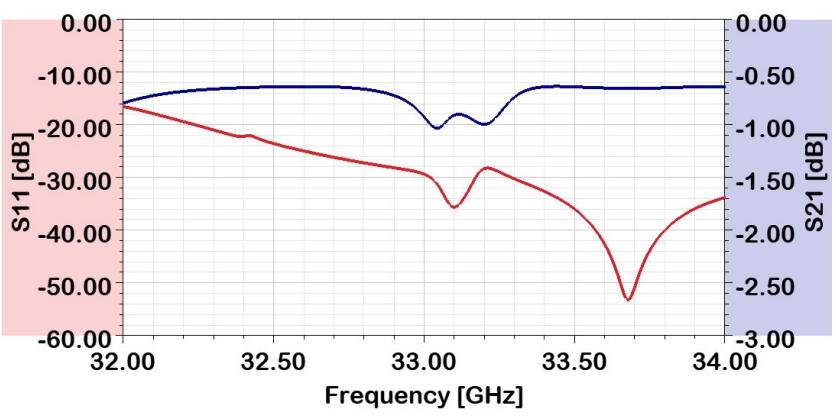

FIGURE 7. $\mathrm{S}_{11}$ (red) and $\mathrm{S}_{21}$ (blue) of the complete structure (32-34 GHz)

\section{COMPLETE STRUCTURE}

After the design and simulation phase has been terminated, the structural part of the project started, including the 3D CAD modeling of the electronic components and the realization of the mechanical frame. The solid-state MMIC power amplifiers stand on a $1.5 \mathrm{~mm}$ thick copper carrier, in 
order to allow the correct disposal of the heat. Cu-Mo heat spreaders are used below MMICs, to assure proper compensation between coefficients of thermal expansion of copper carrier and MMIC. Two stacks of capacitors were arranged along the two long sides of the MMIC taken as an example for the dimensioning (Qorvo TGA2222) and golden feedthroughs carry the power supply. All these parts were enclosed within an aluminum module divided into two shells and tightened with four M 1.4 screws; another quartet of the same screws was used to anchor the module to the waveguide. For this section (waveguide), starting from a solid aluminum parallelepiped, the so-called EDM (Electrical Discharge Machining) process can be adopted in order to obtain the shape of the waveguide polarizer, while the holes can be made with a milling cutter. Finally, a heat sink system was designed. The spatial power combiner can now be cooled by a cold plate connected to a reserve of liquid nitrogen, moreover the connection surface between cold plate and heat sink can be any of the six available. In figure 8 the whole structure can be appreciate; on the left side, we can see, from left to right, the input waveguide port in WR28, then the four amplification modules and finally the output port in the same WR28 waveguide. On the right side, a picture of the SPC supplemented by the heat sink is depicted.

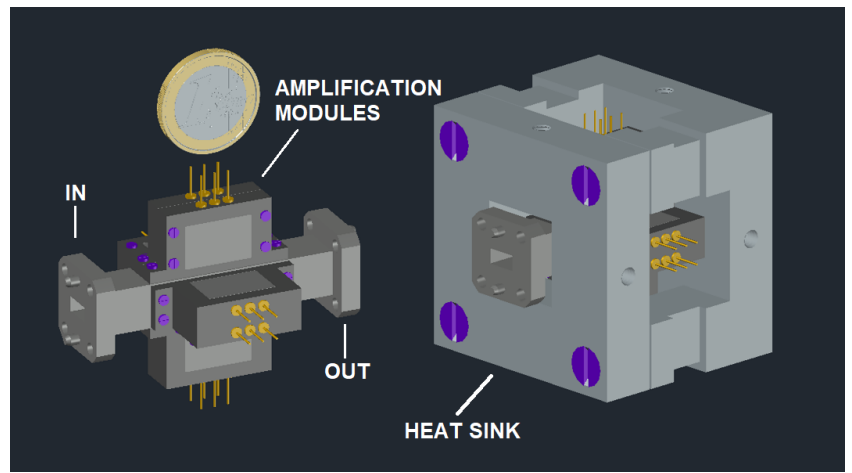

FIGURE 8. Drawings of the innovative SPC. Note the SPC size respect 1 Euro coin.

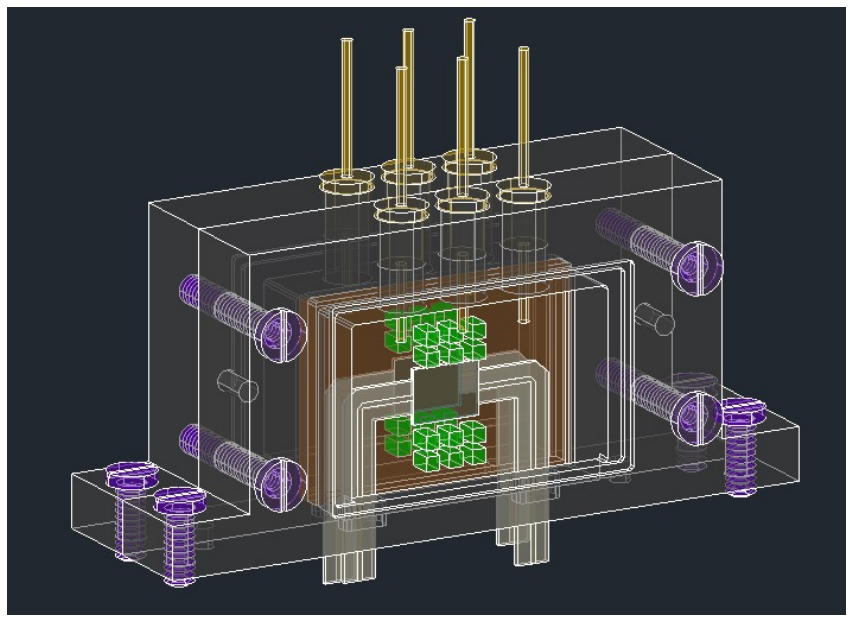

FIGURE 9. An x-ray view of the amplification module.

\section{MULTIPHYSICS SIMULATIONS}

Since the possible applications of this structure may vary from avionics to satellite, further multiphysics analyses are essential in order to fully characterize the structure from an engineering viewpoint. Two different types of these simulations were carried out, both using the CAD software COMSOL $4.0[17,18]$, specifically thermal and structural ones. For what concerns the thermal simulations, the first step is to simplify the structure (removing the screws and more) in order to achieve convergence; then a schematization of the power distribution within the volume of the MMIC power amplifiers is fundamental. In the specific case of the Qorvo MMIC type TGA2222, there are two stages of amplification, a driver one and a final stage. In order to take advantage of the symmetry function provided by the software, the total power were equally divided between driver and final stage, which were represented with two parallelepipeds placed inside the volume of the solid state amplifier; once that was done, the complete structure (SPC and Heat Sink) was cut in half and the symmetry function could be applied.

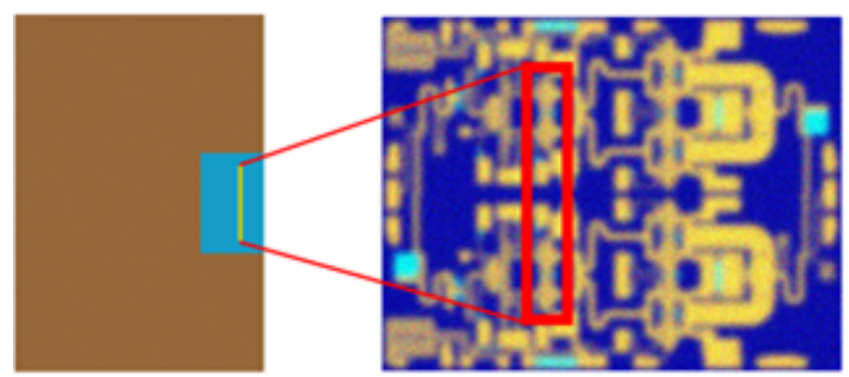

FIGURE 10. Graphic Schematization of the MMIC FET channels, whose transverse length is $2 \mathrm{~mm}$.

Supposing to connect the cold plate on the upper face of the Heat Sink, the heat distribution will be that shown in fig. 11; being placed at different distances from the cold plate, vertical and horizontal solid state amplifiers experience different temperatures which are shown in fig. 12.

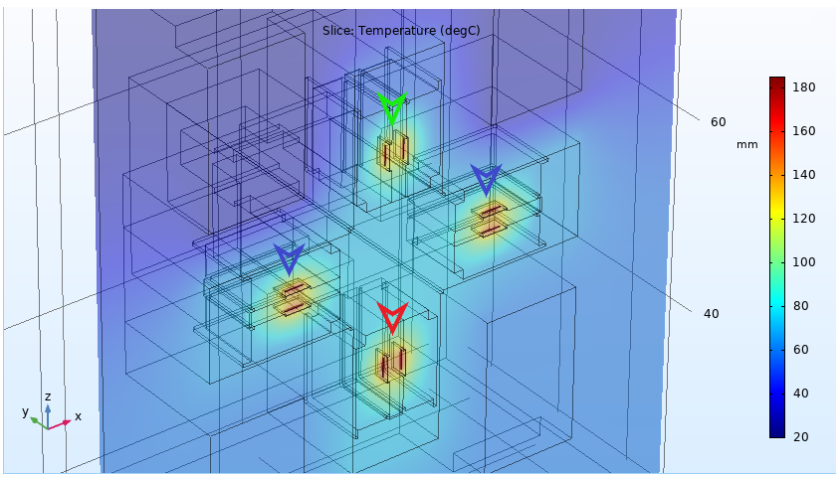

FIGURE 11. Heat Distribution and Maximum Temperatures in ${ }^{\circ} \mathrm{C}$. The markers are linked to the curves depicted in figure 12 , according to their colors. 


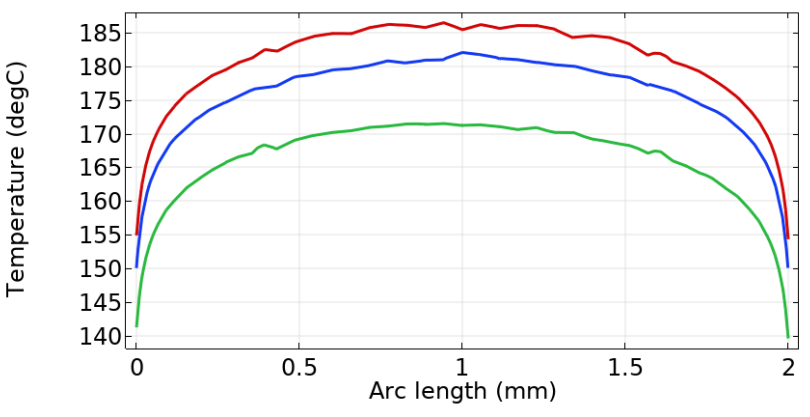

FIGURE 12. Temperature of the Horizontal (blue) and Vertical (green, red) Solid State Amplifiers.

Of course, if needed the cold plate can be placed at bottom surface of the heat sink as well, resulting in equal temperature for bottom and top MMICs.

Regarding the structural simulations, the same simplified structure of the thermal simulations was used, but in this case no symmetry could be applied, therefore the entire 3D design of the structure was considered. The goal of this design phase was to make sure that all mechanical resonance frequencies are as much as possible above $10 \mathrm{KHz}$. This is in fact the maximum frequency at which mechanical stress tests are carried out on satellite modules, and occurs during the socalled pyrotechnic shock in which the module must resist to shock waves caused by explosives in the launch phase.

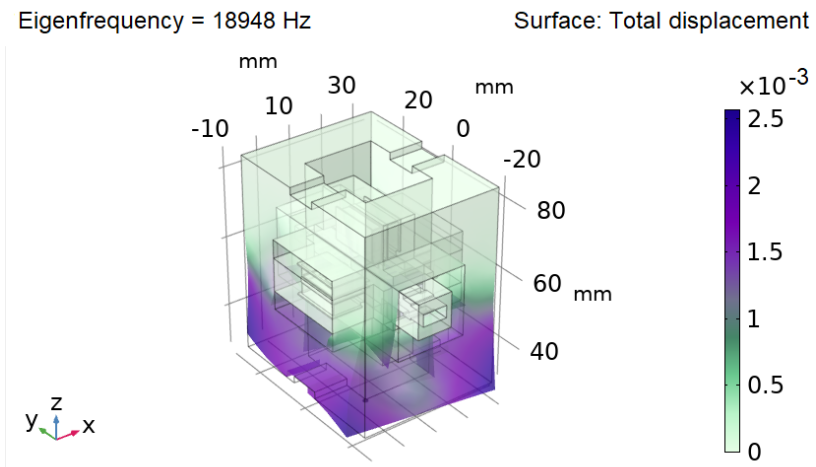

FIGURE 13. Mechanical Deformations at First Eigenfrequency $(18 \mathrm{KHz})$

$$
\text { Eigenfrequency }=57216 \mathrm{~Hz} \quad \text { Surface: } \text { Total displacement }(\mathrm{mm})
$$

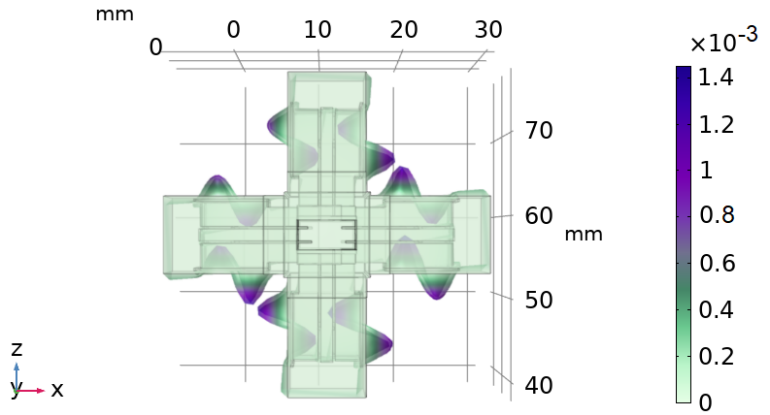

FIGURE 14. Mechanical Deformations at one of the Eigenfrequencies (57 $\mathrm{KHz})$

As shown in figure 13, the first eigenfrequency starts beyond $18 \mathrm{KHz}$, then for a value almost double compared to the lower bound of $10 \mathrm{KHz}$. This shows that the structure is also able to pass structural qualification tests in case of satellite applications and can therefore be installed on any flying station, either avionic or space one.

\section{CONCLUSIONS}

A completely innovative spatial power combiner has been designed, exploiting the electromagnetic field rotation introduced in a waveguide polarizer and conveying the transit power by means of transversal probes made by microstrips. Electromagnetic and Multiphysics Simulations show excellent performances, resulting in a virtual prototype ready to be realized with success. A new goal has been reached, achieving a power division by eight completely carried out in space, with a power-combining efficiency close to $80 \%$ in the $32-34 \mathrm{GHz}$ band. Using eight solid state power amplifiers with RF power of $10 \mathrm{~W}$, the combined output power of the signal reaches $65 \mathrm{~W}$ also not exceeding the constraints on heat dissipation, as shown by multiphysics simulations. This remarkable result, though, lies in the outstanding power density of this structure; great integration capabilities and small dimensions allowed to reach 4200 $\mathrm{mW} / \mathrm{cm}^{3}$ of volume power density and $1450 \mathrm{~W} / \mathrm{Kg}$ of mass power density, exceptional values for millimeter-wave electronics.

\section{REFERENCE}

[1] D. Passi, A. Leggieri, F. Di Paolo, M. Bartocci, A. Tafuto, "Spatial Power Combiner Technology", in PIERS Proc., Czech Republic, 2015, pp. 932-938.

[2] A. Leggieri, D. Passi, F. Di Paolo, G. Orengo, "The Squarax Spatial Power Combiner", Progress In Electromagnetics Research (PIER) C, Vol.45, October 2013, p. 43-55.

[3] D. Passi, A. Leggieri, R. Citroni, F. Di Paolo, "New SixWay Waveguide to Microstrip Transition applied in X Band Spatial Power Combiner", in Proc. of $4^{\text {th }}$ Advanced Electromagnetics Symposium, Malaga, Spain, 26-28 Jul. 2016, pp. 264-267.

[4] D. Passi, A. Leggieri, F. Di Paolo, M. Bartocci, A Tafuto and A. Manna, "High Efficiency Ka-Band Spatial Power Combiner", Advanced Electromagnetics, vol. 3, no. 2, pp. 10-15, 2014.

[5] D. Passi, A. Leggieri, R. Citroni, F. Di Paolo, "Broadband TE10 to TE20 Mode Transformer for X Band", in Proc. Of $4^{\text {th }}$ Advanced Electromagnetics Symposium, Malaga, Spain, 26-28 Jul. 2016 pp. 261264.

[6] D. Passi, A. Leggieri, A. Mattioni, F. Di Paolo, M D'Antoni, M. Bartocci, E. Ciacia, A. Tafuto, "Small Size, High Power Density, Solid State Amplifiers for Space Application", International Symposium on Networks, Computers and Comunications (ISNCC), 2018.

[7] A. Leggieri, D. Passi, F. Di Paolo, G. Saggio, "Multiphysics Design of a Spatial Power Combiner Predisposed for Thermo-mechanically Affected Operation", Journal of Electromagnetic Waves and 
Applications, Taylor \& Francis, Vol. 28, Issue 17, September 2014.

[8] L. A. Rud and K. S. Shpachenko, "Polarizers on Section of Square Waveguides with inner corner Ridges", 2011 VIII International Conference on Antenna Theory and Techniques, September 20-23, Kyiv, Ukraine.

[9] Kang Yin, JinPin Xu and ZhengHua Chen, "A Full Kaband Waveguide-based Spatial Power-combining Amplifier Using E-Plane Anti-Phase Probes", State Key Lab. of Millimeter Waves, Southeast University, Nanjing, JiangSu.

[10] Yi-Hong Zhou, Jia-Yin Li, Bo Zhao and Hai-Yang Wang, "A Ka-Band Power Amplifier Based on Doubleprobe Microstrip to Waveguide Transition", Progress In Electromagnetics Research Symposium Procedings, Xi'an, China, March 22-26, 2010.

[11] Zenon R. Szezepaniak, "Broadband Waveguide Power Splitter for X-band Solid-state Power Amplifiers", Proceedings of Asia-Pacific Microwave Conference 2007, Telecomunications Research Institute Poligonowa 30, Warsaw, Poland.

[12] https://www.enginsoft.com/solutions/ansys-hfss.html

[13] Xing Wang, Xiaodong Huang and Xiuhua Jin, "Novel Square/Rectanngle Waveguide Septum Polarizer", IEEE International Conference on Ubiquitous Wireless Broadband (ICUWB), 2016.

[14] J. Bornermann, V. A. Labay, "Ridge Waveguide Polarizer with Finite and Stepped-Thickness Septum", IEEE Transactions on Microwave Theory and Techniques, Vol. 43, No 8, August 1995.

[15] Xiaoqiang Xie, Xiang Zhao and Xiao Liu, "A Waveguide-Based Spatial Power Combining Module at Higher Millimeter-Wave Frequency", Journal of Infrared, Millimeter, and Teraherts Waves, 2013.

[16] D. Passi, A. Leggieri, F. Di Paolo, M. Bartocci, A. Tafuto, "Design of High Power Density Amplifiers: Application to Ka Band", Journal of Infrared, Millimeter, and Terahertz Waves, 2017.

[17] https://www.comsol.com/heat-transfer-module

[18] https://www.comsol.com/structural-mechanics-module 